\title{
Molecular dynamics simulation of small water-binding cavitands
}

\author{
Giovanna Longhi ${ }^{a}$, Fabrizio Fabris ${ }^{\mathrm{b}}$, Cristiano Zonta ${ }^{\mathrm{b}}$, Sandro L. Fornili ${ }^{\mathrm{c}, *}$ \\ a Dipartimento di Scienze Biomediche e Biotecnologie, Università di Brescia, viale Europa 11, I-25123 Brescia, Italy \\ b Dipartimento di Chimica, Università Ca' Foscari di Venezia, Dorsoduro 2137, I-30123 Venezia, Italy

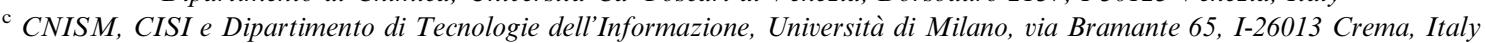

Received 21 February 2006

Available online 7 April 2006

\begin{abstract}
Analyses of 20-ns simulations of aqueous solutions of the recently synthesized cavitand benzotriborneol (BTB) show that this molecule binds a single water molecule within its hydrophilic cavity for an average time interval of ca. $750 \mathrm{ps}$, that is 370 times longer than the permanence time of water around borneol. Moreover, this time becomes three times longer in a $99.8 \%$ (V/V) chloroform-water solution, while it decreases for BTB methylether derivatives in water, becoming 279, 36 or 119 ps when one, two or all three hydroxyl hydrogen atoms are replaced by methyl groups, respectively.
\end{abstract}

(C) 2006 Elsevier B.V. All rights reserved.

\section{Introduction}

The study of water in confined geometry conditions can enlighten properties that are potentially useful to understand its behavior when involved within proteins [1], molecular channels [2], polymers [3] or more complex systems [4].

In the present Letter, we report results obtained by molecular dynamics (MD) simulations concerning the interaction of water with a recently synthesized molecule (benzotriborneol, BTB, see Fig. 1) [5] featuring two cavity-like regions, one of which possesses a hydrophilic character due to the presence of three hydroxyl groups.

The possibility that this host could be a strong water receptor was suggested by the experimental observation [5] that during the purification phase it was difficult to dry this triol with standard anhydrification procedures (e.g., magnesium sulphate, reduced pressure). As the bottom trace of Fig. 2 shows, residual water was still present after this treatment. The compound was finally dried by azeotropic removal of water in boiling toluene (Fig. 2,

\footnotetext{
* Corresponding author. Fax: +3902 50330010.

E-mail address: fornili@dti.unimi.it (S.L. Fornili).
}

top trace). These findings prompted us to start this simulation work aiming to ascertain whether the BTB stereodynamics is particularly suitable to host a water molecule in its hydrophilic cavity.

Since such a strong interaction with water was not observed for the trimethylether derivative of BTB [5] we also decided to investigate systems in which one or more hydrogen atoms of the BTB hydroxyl groups are replaced by methyl groups. Moreover, in order to simulate experimental conditions similar to those used to obtain the results shown in Fig. 2, a BTB solution in $99.8 \%(\mathrm{~V} / \mathrm{V})$ chloroform-water was also considered.

\section{Computational methods}

BTB and the related molecules examined in this work were fully optimized with Gaussian 98 [6] at RHF/6$31 \mathrm{G}^{*}$ level. At the same level, molecular properties were calculated to evaluate their atomic charges according to the RESP procedure [7]. Simulations were performed using the Amber ff99 force field [8] and the SANDER program [9] with truncated octahedral periodic boundaries. The temperature was controlled according to the Berendsen coupling algorithm [10] with $\tau_{T}=1 \mathrm{ps}$, and $\tau_{P}=1 \mathrm{ps}$ was 


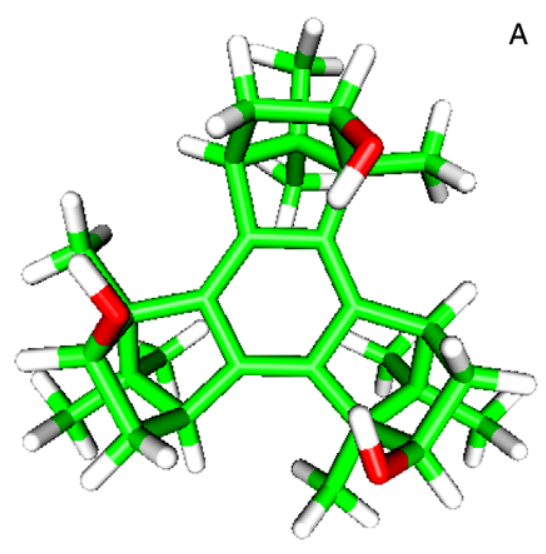

A

B
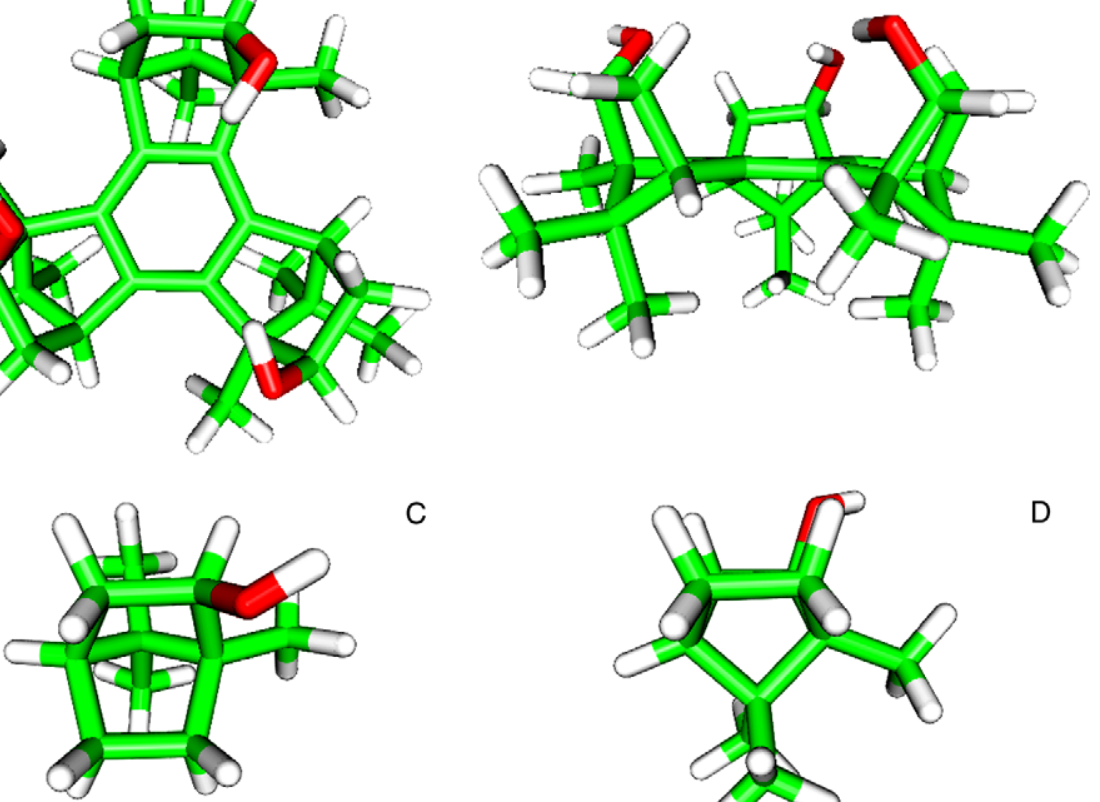

C

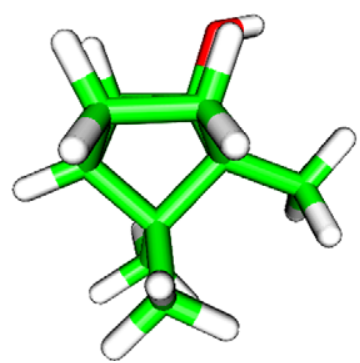

Fig. 1. Stick representation of benzotriborneol (BTB) and borneol molecules. Upper row: top (A) and front (B) views of BTB. Lower row: top (C) and front (D) views of the borneol. The top views evidence the hydroxyl groups of molecules.

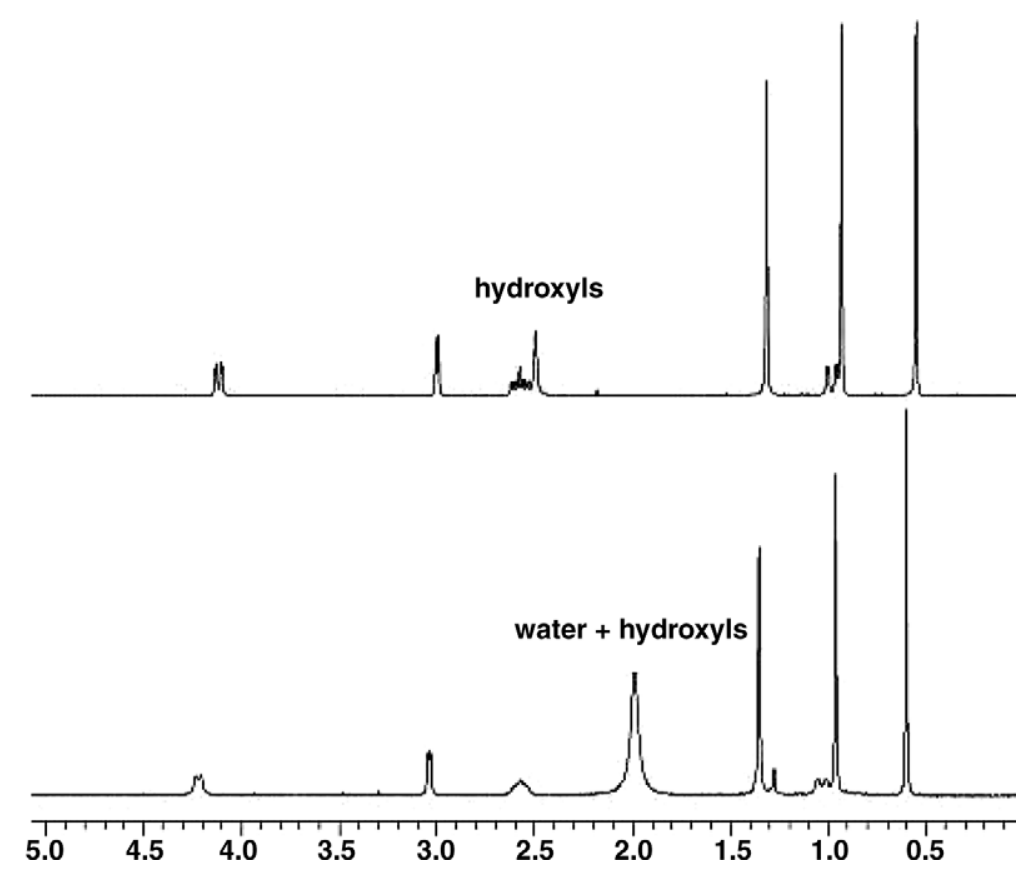

Fig. 2. Top trace: ${ }^{1} \mathrm{H}$ NMR spectrum of BTB in chloroform after azeotropic removal of water. Bottom trace: ${ }^{1} \mathrm{H}$ NMR spectrum of BTB in chloroform solution in the presence of residual water after standard drying procedure.

used for the pressure control. A cutoff of $9 \AA$ and Ewald sums were adopted for the nonbonded interactions. SHAKE [10] kept constrained bonds involving hydrogen atoms. NPT ( $T=298 \mathrm{~K}, P=1 \mathrm{bar}$ ) simulations were performed with 1-fs time step. Water was modeled according to the TIP3P scheme [11], and the geometry and atomic charges of chloroform were assumed as in Ref. [12]. System configurations were stored on disk every $0.5 \mathrm{ps}$ for off-line statistical analyses based on home-made programs, and PTRAJ and ANAL modules of the AMBER7 package. Graphical visualization was performed using gOpenMol [13]. 


\section{Results and discussion}

For each system listed in Table 1, two NPT 20-ns simulations have been performed. The system labeled as $B T B$ consists of one BTB molecule and 1055 water molecules, while the $B T B$-chl system includes one BTB, 10 water and 1065 chloroform molecules. The BTB-1m, BTB-2m and $B T B-3 m$ systems include one mono-, di- or tri-methylether BTB molecule (namely, a BTB molecule with one, two or three hydroxyl hydrogen atoms replaced by methyl groups) and 1125, 1251 and 1127 water molecules, respectively. For comparison, a system has also been considered consisting of 612 waters and one borneol molecule, which can be considered a building block of the BTB molecule (Fig. 1).

In order to get a general insight into the solute hydration properties, we have evaluated the space distribution functions (SDFs) of the oxygen atoms of the water molecules residing in the solute first hydration shell (i.e., within $3.5 \AA$ from the solute atoms) according to the following expression [14]:

$g(\mathbf{r})=\rho(\mathbf{r}) / \rho_{0}$ where $\rho(\mathbf{r})$ indicates the number density of the water oxygen atoms at the position $\mathbf{r}$ and $\rho_{0}$ its average within the simulation box. The position vector $\mathbf{r}$ is defined in a coordinate system attached to the solute molecule.

In Fig. 3, we report the contour map corresponding to the level $g(\mathbf{r})=2.2$ of the SDF for the BTB system. As expected, the solute hydration is more relevant in the hydrophilic side of the molecule.

The contour maps reported in Fig. 4 show the SDFs for the $B T B, B T B-1 m, B T B-2 m$ and $B T B-3 m$ systems represented at the $g(\mathbf{r})=5.0$ level to limit the visualization to larger SDF values. One may notice that the common feature of all these systems is the prominent presence of water at the center of the hydrophilic cavity. Indeed, in this space region statistical analysis shows that the number density $\rho(\mathbf{r})$ is at least 30 times larger than $\rho_{0}$.

Fig. 5 shows that for all systems the highest SDF values correspond to the presence in the hydrophilic cavity of the solute molecules of a single water molecule, whose average permanence time $T$ values within the cavity are reported in Table 1 . They are evaluated by averaging the consecutive time intervals that a given water molecule remains in the cavity. The time resolution of this analysis is 1 ps. For com-

Table 1

$\mathrm{T}$ is the average time spent by the water molecule bound within the solute hydrophilic cavity (i.e., the space region delimited by the solute oxygen atoms) $E_{\mathrm{SW}}$ is the average interaction energy between solute and bound water molecule, on the average forming $N_{\mathrm{HB}}$ hydrogen bonds with the solute. $D_{\mathrm{CM}-\mathrm{OW}}$ and $D_{\mathrm{CM}-\mathrm{HW}}$ represent the average distance (and in parentheses their standard deviations) between the center of mass of the solute oxygen atoms and the oxygen and hydrogen atoms of the bound water molecule, respectively. $N_{\mathrm{FSW}}$ is the average number of the first-shell water molecules (i. e., within $3.5 \AA$ from the atoms of the solute), some of which are close to the solute oxygen atoms (data in parentheses). The simulated systems are described in the text

\begin{tabular}{|c|c|c|c|c|c|c|}
\hline Systems & $T(\mathrm{ps})$ & $E_{\mathrm{SW}}(\mathrm{kcal} / \mathrm{mol})$ & $D_{\text {CM-OW }}(\AA)$ & $D_{\mathrm{CM}-\mathrm{HW}}(\AA)$ & $N_{\mathrm{HB}}{ }^{\mathrm{a}}$ & $N_{\text {FSW }}$ \\
\hline$B T B$ & 747 & -13.5 & $0.8(0.3)$ & $1.1(0.3)$ & 2.3 & $52.1(9.6)$ \\
\hline$B T B-c h l$ & 2226 & -13.7 & $0.7(0.2)$ & $1.1(0.3)$ & 2.3 & $5.3(4.5)$ \\
\hline$B T B-1 m$ & 279 & -11.0 & $0.8(0.4)$ & $1.1(0.4)$ & 2.0 & $54.2(7.7)$ \\
\hline$B T B-2 m$ & 36 & -7.8 & $1.1(0.8)$ & $1.2(0.8)$ & 1.3 & $56.3(5.8)$ \\
\hline$B T B-3 m$ & 119 & -7.4 & $1.3(0.5)$ & $1.1(0.5)$ & 0.6 & $58.4(4.3)$ \\
\hline Borneol & 2 & -4.5 & $2.8(0.2)$ & $2.6(0.7)$ & 0.8 & $29.2(4.1)$ \\
\hline
\end{tabular}

${ }^{a}$ Hydrogen bond definition: distance $r_{\text {Овтв-Оw }} \leqslant 3.5 \AA$; angle $\theta_{\text {Овтв-Нw-Оw }}$ or $\theta_{\text {Овтв-Нвтв-Оw }} \geqslant 150^{\circ}[16]$.
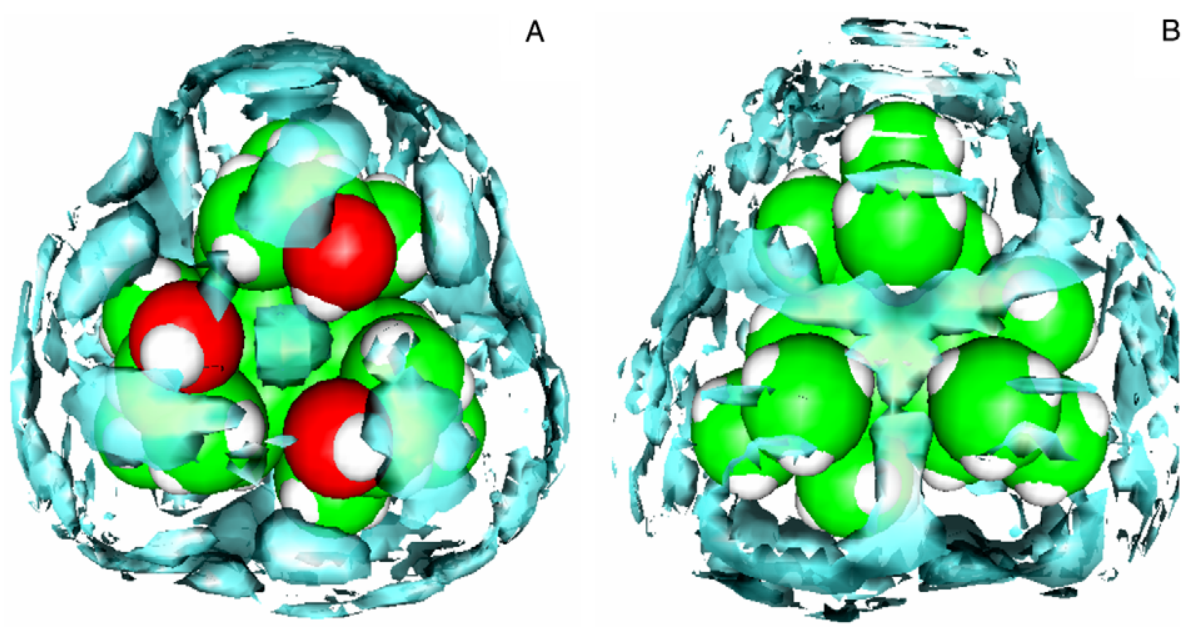

Fig. 3. Spatial distribution functions (SDFs), $g(\mathbf{r})$, of the water oxygen atoms surrounding the BTB molecule: top (A) and bottom (B) views. The isosurfaces correspond to the level $g(\mathbf{r})=2.2$. 

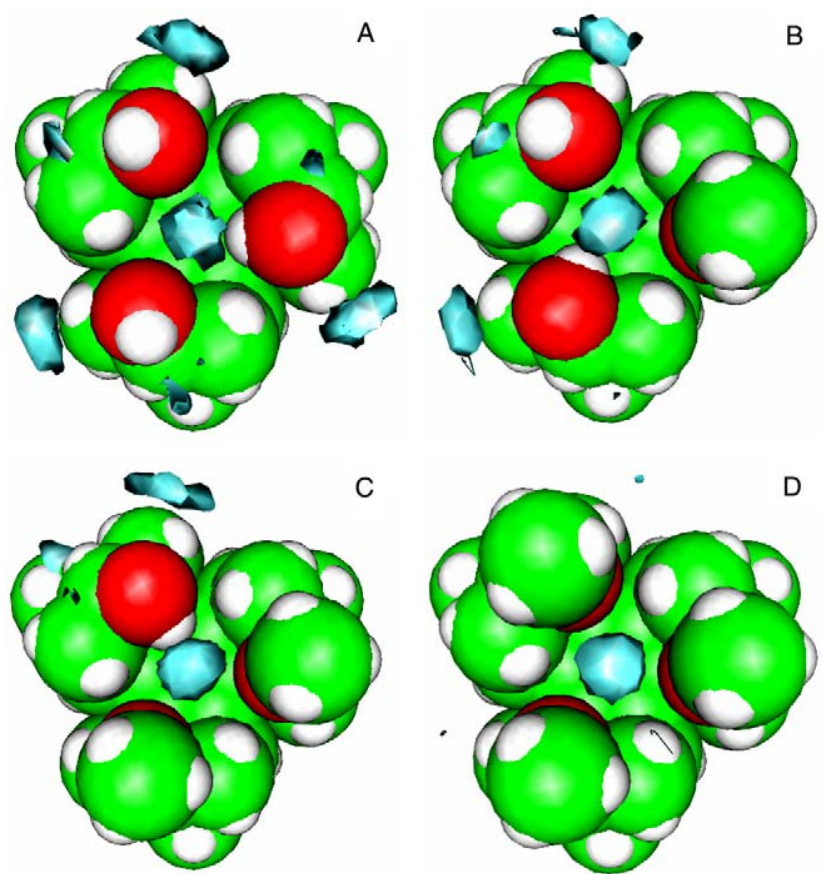

,

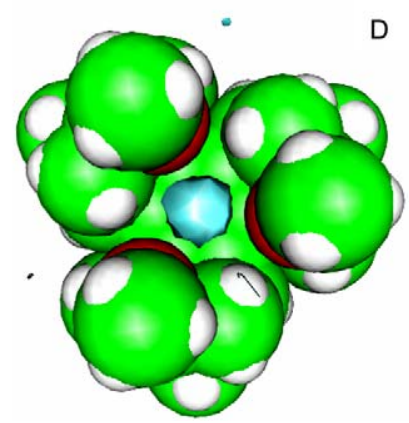

Fig. 4. Top views of the spatial distribution functions of the water oxygen atoms surrounding different solutes. Upper row: BTB (A), BTB-1m (B). Lower row: BTB-2m (C) and BTB-3m (D). BTB- $x$ m indicates a molecule obtained by BTB by replacing $\boldsymbol{x}$ hydroxyl hydrogen atoms with methyl groups. The isosurfaces correspond to the level $g(\mathbf{r})=5.0$.

parison, a similar analysis has been performed for borneol by averaging successive time intervals a given water molecule keeps being the closest to the hydroxyl group. From Table 1, one can see that the $T$ value for BTB is ca. 370 times larger than that for borneol. It is also worth mentioning that the analysis of trajectories of aqueous solutions of trimethylamine- $N$-oxide (TMAO) and of tert-butyl alcohol from previous simulations [15] yields $T$ values similar to that for borneol.

The presence of chloroform as a co-solvent considerably lengthens the corresponding $T$ value which becomes three times larger than that for the $B T B$ system. This is paral- leled by the number of water molecules within the solute first hydration shell, $N_{\mathrm{FSW}}$, reported in Table 1 . Indeed, on the average almost half of the water molecules of the $B T B$-chl system remain in the neighborhood of the hydrophilic moiety of the solute rather than being uniformly distributed within the simulation box.

Table 1 shows also that the $T$ value corresponding to the $B T B-1 m$ system becomes ca. 2.7 times smaller than that for the $B T B$ system, as a result of the replacement of one hydroxyl hydrogen atom with a hydrophobic methyl group. The replacement of a second hydroxyl hydrogen atom $(B T B-2 m$ system) induces a further strong decrease of the $T$ value, which becomes more than 20 times smaller than that corresponding to the $B T B$ system.

These findings can be explained considering that the cavity hydrophilicity decreases with increasing the number of methyl groups. However, if the last hydroxyl hydrogen atom is replaced by a methyl group, passing from the $B T B-2 m$ system to the $B T B-3 m$ system, a threefold increase in the $T$ value is observed. To enlighten this point, the trajectories have been statistically analyzed to evaluate energetic and geometric properties of the water molecules residing within the hydrophilic molecular cavity. From Table 1, we can see that the average values of the interaction energy, $E_{\mathrm{Sw}}$, of such molecules with the solutes decreases monotonically with the number of methyl groups. The same behavior is also present for the number of hydrogen bonds, $N_{\mathrm{HB}}$, formed by the water inside the cavity and the hydrophilic groups of the solutes. Therefore, neither of these quantities is useful to explain the $T$ minimum corresponding to the $B T B-2 m$ system.

A possible explanation is instead suggested by examining the distance values from the center of mass of the oxygen atoms of the solutes and the oxygen and hydrogen atoms of the water inside the cavity, $D_{\text {CM-Ow }}$ and $D_{\mathrm{CM}-\mathrm{HW}}$, respectively. One can see that for all systems except $B T B-3 m$, the water oxygen atom is on the average closer to the center of mass of the solute oxygen atoms than the water hydrogen atoms. This suggests that for all systems except $B T B-3 m$ the solute hydroxyl hydrogens are
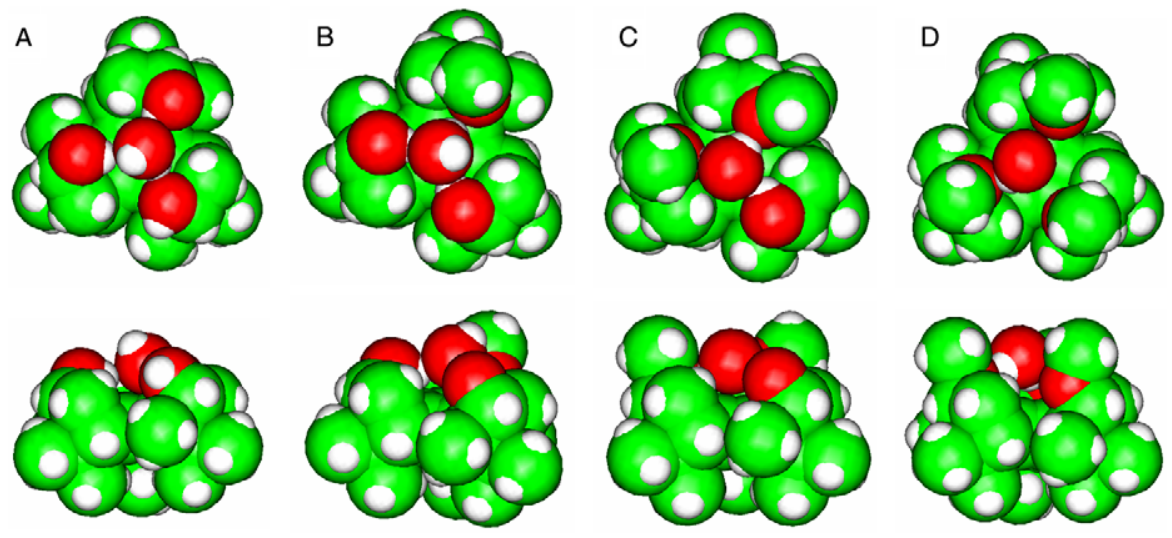

Fig. 5. Top (upper row) and front (lower row) views of the simulated solutes including the water molecule bound within the hydrophilic cavity: (A) BTB, (B) BTB-1m, (C) BTB-2m, (D) BTB-3m. Data are taken from statistically representative snapshots. 
more effective than the oxygen atoms in attracting the water molecule inside the cavity. We also observe that the smallest value of the difference between $D_{\mathrm{CM} \text {-Ow }}$ and $D_{\mathrm{CM}-\mathrm{HW}}$ is for $B T B-2 m$, suggesting that the average orientation of the water molecule inside the cavity is in this case not as firm as for the other systems. It seems then reasonable to conclude that in these conditions the water molecule inside the cavity is more prone to leave the solute, although it remains within it for a time interval which is almost 20 times longer than around borneol. The propensity of water to leave the cavity in $B T B-2 m$ case is also indicated by the rather high value of standard deviation of both $D_{\mathrm{CM} \text {-Ow }}$ and $D_{\mathrm{CM} \text {-Hw }}$ reported in Table 1 .

\section{Conclusions}

Results obtained by simulating aqueous solutions of the recently synthesized cavitand molecule benzotriborneol (BTB) show that the latter binds one water molecule inside its hydrophilic cavity for a time interval of $747 \mathrm{ps}$, as averaged over two 20-ns MD simulations. This time is ca. two orders of magnitude longer than the time the closest water molecules spend in the neighborhood of a borneol molecule, which may be considered a component of the BTB molecule.

When the BTB molecule is solvated by $99.8 \%(\mathrm{~V} / \mathrm{V})$ chloroform-water solvent, such as to mimic conditions suitable for ${ }^{1} \mathrm{H}$ NMR spectroscopy, the average permanence time of water within the BTB cavity becomes almost three times longer. Instead, if aqueous solutions are considered including a BTB molecule with one, two or all three hydrogen atoms of its hydroxyl groups replaced by methyl groups, the permanence time is reduced approximately by a factor of 3, 21 or 6, respectively. Analyses of energetic and structural properties of these systems suggest that the minimum value occurring when two hydroxyl hydrogen atoms are replaced by methyl groups could be induced by the fluc- tuating action exerted by the residual hydroxyl hydrogen atoms on the water molecule bound in the BTB hydrophilic cavity.

\section{Acknowledgement}

We thank Sergio Abbate for helpful discussions, and Davide Rebeccani, Massimo Fierro and Paolo Ferri for technical assistance. General indirect support has been provided by FIRST funds (Milan) and two PRIN national framework programs (Venice and Brescia).

\section{References}

[1] A. Purkiss, S. Skoulakis, J.M. Goodfellow, Phil. Trans. R. Soc. Lond. A 359 (2001) 1515 .

[2] S.M. Saparov, P. Pohl, Proc. Natl. Acad. Sci. USA 101 (2004) 4805.

[3] B. Sreenivasulu, J.J. Vittal, Angew. Chem., Int. Ed. 43 (2004) 5769.

[4] M.-C. Belissent-Funel, C.R. Geoscience 337 (2005) 173.

[5] F. Fabris, L. Bellotto, O. De Lucchi, Tetrahedron Lett. 44 (2003) 1211.

[6] M.J. Frisch et al., Gaussian 98, Gaussian Inc., Pittsburgh, PA, 1998.

[7] C.I. Bayly, P. Cieplak, W.D. Cornell, P.A. Kollman, J. Phys. Chem. 97 (1993) 10269.

[8] J. Wang, P. Cieplak, P.A. Kollman, J. Comput. Chem. 21 (2000) 1049.

[9] D.A. Case et al., Amber 7, University of California, San Francisco, 2002.

[10] M.P. Allen, D.J. Tildesley, Computer Simulation of Liquids, Clarendon Press, Oxford, 1987.

[11] W.L. Jorgensen, J. Chandrasekhar, D. Madura, R.W. Impey, M.L. Kein, J. Chem. Phys. 79 (1983) 926.

[12] P. Cieplak, J. Caldwell, P. Kollman, J. Comput. Chem. 22 (2001) 1048.

[13] D.L. Bergman, L. Laaksonen, A. Laaksonen, J. Mol. Graph. Model. 15 (1997) 301.

[14] I.M. Svishchev, P.G. Kusalik, J. Chem. Phys. 99 (1993) 3049.

[15] A. Fornili, M. Civera, M. Sironi, S.L. Fornili, Phys. Chem. Chem. Phys. 5 (2003) 4905.

[16] H. Xu, B.J. Berne, J. Phys. Chem. B 105 (2001) 386. 